\title{
Steady-State Visual Evoked Potentials and Phase Synchronization in Migraine Patients
}

\author{
L. Angelini, ${ }^{1,2,3}$ M. De Tommaso, ${ }^{1,4}$ M. Guido, ${ }^{1}$ K. Hu, ${ }^{5}$ P. Ch. Ivanov, ${ }^{5,6}$ D. Marinazzo, ${ }^{1}$ G. Nardulli, ${ }^{1,2,3}$ L. Nitti, ${ }^{1,7,3}$ \\ M. Pellicoro, ${ }^{1,2,3}$ C. Pierro, ${ }^{1}$ and S. Stramaglia ${ }^{1,2,3}$ \\ ${ }^{1}$ TIRES: Center of Innovative Technologies for Signal Detection and Processing, University of Bari, Italy \\ ${ }^{2}$ Physics Department, University of Bari, Italy \\ ${ }^{3}$ Istituto Nazionale di Fisica Nucleare, Sezione di Bari, Italy \\ ${ }^{4}$ Department of Neurological and Psychiatric Sciences, University of Bari, Italy \\ ${ }^{5}$ Center for Polymer Studies and Department of Physics, Boston University, Boston, Massachusetts, USA \\ ${ }^{6}$ Institute of Solid State Physics, Bulgarian Academy of Sciences, 1784 Sofia, Bulgaria \\ ${ }^{7}$ D.E.T.O., University of Bari, Italy \\ (Received 8 October 2003; revised manuscript received 5 April 2004; published 15 July 2004)
}

\begin{abstract}
We investigate phase synchronization in EEG recordings from migraine patients. We use the analytic signal technique, based on the Hilbert transform, and find that migraine brains are characterized by enhanced alpha band phase synchronization in the presence of visual stimuli. Our findings show that migraine patients have an overactive regulatory mechanism that renders them more sensitive to external stimuli.
\end{abstract}

DOI: 10.1103/PhysRevLett.93.038103

Phase synchronization, a concept introduced in the field of nonlinear dynamics [1], provides a measure of synchronization alternative to conventional linear approaches. It may be useful to study electroencephalographic (EEG) signals, where synchronization phenomena are expected to play a major role in establishing the communication between different regions of the brain [2]. A migraine is an incapacitating disorder of neurovascular origin, which consists of attacks of headaches, accompanied by autonomic and possibly neurological symptoms. It is estimated that in the United States, 5\% of the general population suffer at least 18 days of migraine headaches a year, and more than $1 \%$ have at least 1 day of migraine a week [3]. In spite of much research, there are still many unresolved issues in the pathophysiology of migraine. There is a tendency to believe that migraine starts with an underlying central nervous system disorder, which, when triggered by various stimuli, sets off a chain of neurologic and biochemical events, some of which subsequently affect the brain's vascular system. No experimental model fully explains the migraine process [4]. A wide range of events and conditions can alter conditions in the brain that bring on nerve excitation and trigger migraines. They include emotional stress, intense physical exertion, abrupt weather changes, flickering lights, and many others. The question we address here is the following: How does the response of migraine patients to such events differ from the response of healthy subjects? To address this problem, we investigate synchronization phenomena in electroencephalograms (EEGs) recorded from migraine patients during repetitive visual stimuli (steady-state visual evoked potentials, SVEPs [5]), and study how synchronization between responses from different brain regions varies in the presence of such external stimuli (i.e., while the brain is processing exter-
PACS numbers: 87.19.La, 05.45.Xt, 05.45.Tp, 42.66.Lc

nal information). We find that migraine brains show increased alpha band phase synchronization, while healthy subjects show a decreased one. Our results suggest that migraine patients have an overactive regulatory mechanism, prone to instability, which renders them more sensitive to environmental factors.

Our data are as follows. EEG is recorded from 15 patients affected by migraine without aura [6], in the presence of visual stimuli. During the acquisition, flash stimuli are presented to the subjects repetitively at a rate of 3-6-9-12-15-18-21-24-27 Hz. The mean age of patients is 38.7 years (range 24-48 years). Each frequency of stimulation is delivered by a flash settled at a luminance of $0.2 \mathrm{~J}$ for at least $20 \mathrm{sec}$; an interval of $20 \mathrm{sec}$ is interposed between the different trains of stimulation. EEG data are recorded by 18 scalp electrodes, placed according to the international $10-20$ system, referred to $\mathrm{CZ}$ derivation. Impedance is settled below $5 \mathrm{k} \Omega$, EEG is digitally filtered off line by means of a digital filter with a bandpass of $0.3-30 \mathrm{~Hz}$; the sampling rate is $128 \mathrm{~Hz}$. Examples of EEG signals are shown in Fig. 1: also spontaneous EEG (i.e., in the absence of stimuli) is recorded for all patients. All patients are in the interictal state, the time from the end of the last attack being at least $72 \mathrm{~h}$. Moreover, EEG data from 15 healthy subjects (ages ranging from 22 to 45 years) are measured so as to have a control group.

Next we describe our findings. EEG signals are filtered in the alpha band $(8-12.5 \mathrm{~Hz})$. Instantaneous phases are evaluated for all signals; the synchronization index $\rho_{1,1}$, based on the Hilbert transform [7] is then evaluated for all pairs of electrodes, for all 30 subjects and for all frequencies of the flash stimuli. These indexes are subsequently averaged over all the possible pairs of sensors, for each subject both in the presence of stimuli and 


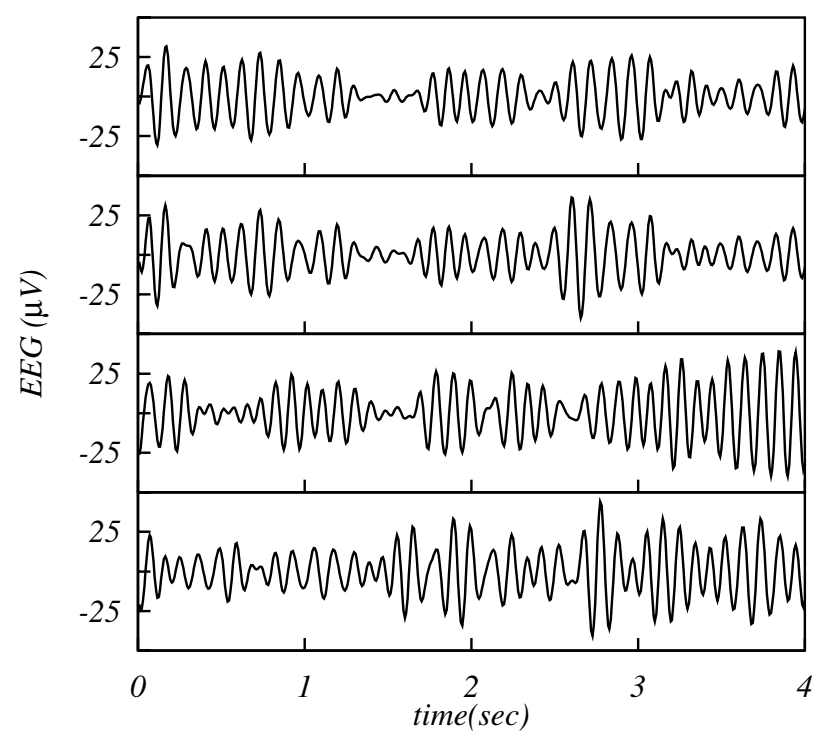

FIG. 1. Examples of EEG signals from a migraine patient. From top to bottom: the signal from $\mathrm{F} 1$ electrode under $9 \mathrm{~Hz}$ flash stimulation; from $\mathrm{F} 2,9 \mathrm{~Hz}$ stimuli; from $\mathrm{F} 1$ in spontaneous conditions; from F2, spontaneously. The full records are $40 \mathrm{sec}$ long, only $4 \mathrm{sec}$ segments are shown. Signals are filtered in the alpha band.

in spontaneous conditions. These mean values do not separate patients from healthy subjects; what emerges as correlated with the migraine pathology is the ratio $\gamma=$ $\rho_{1,1}^{f} / \rho_{1,1}^{s p}$, where $\rho_{1,1}^{f}$ is the mean phase synchronization in the presence of flash stimuli, whereas $\rho_{1,1}^{s p}$ is the mean spontaneous phase synchronization. This ratio measures how phase synchronization varies, in the presence of the stimuli, with respect to basal conditions, i.e., the neat effect of the stimulus. Our supervised analysis (hypothesis testing) shows that the index $\Gamma=\ln (\gamma)$ [8] separates the class of patients and the class of controls for stimulus frequencies of 9,24,27 Hz. For each of the nine flash stimuli frequencies $\omega$, we apply the paired $t$ test to evaluate the probability $P_{\omega}$ that indexes $\Gamma s$ were drawn from the same distribution (the null hypothesis); in seven cases out of nine this probability is less than 0.05, the standard value used in literature to reject the null hypothesis. However, here we deal with multiple comparisons. To control the number of false positives, we use the false discovery rate (FDR) method [9]. This procedure selects the stimuli frequencies $9-24-27 \mathrm{~Hz}$ as separating patients from controls [see Fig. 2(a)], with the expected fraction of false positive 0.05 . The same frequencies (9$24-27 \mathrm{~Hz}$ ) are selected by use of the standard Bonferroni correction for multiple comparisons [10] as well as by FDR if probabilities are evaluated by the nonparametric Wilcoxon rank sum test [see Fig. 2(b)].

A topographic analysis is also performed, in order to check whether this phenomenon is localized in some cortex region. We evaluate, for each sensor $s, \Gamma_{s}=$ $\ln \left(\left\langle\rho_{1,1}^{f}\right\rangle_{s} /\left\langle\rho_{1,1}^{s p}\right\rangle_{s}\right)$, where $\langle\cdot\rangle_{s}$ means averaging only over
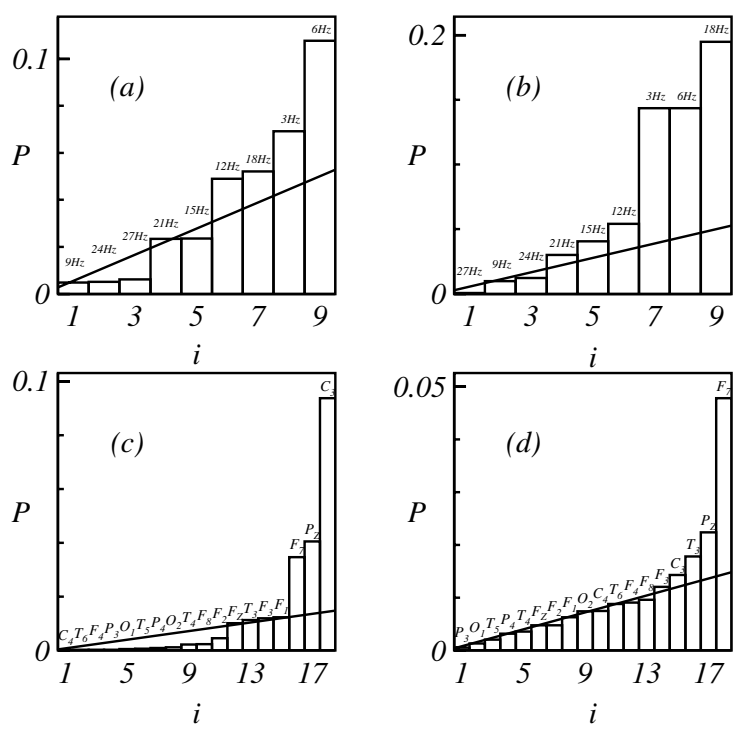

FIG. 2. (a) Application of FDR method. The vertical axis represents the probability that the $30 \Gamma$ values of patients and controls were drawn from the same distribution, according to the paired $t$ test. Frequencies 9-24-27 Hz are selected with false positive rate 0.05 . (b) The probability that the $30 \Gamma$ values of patients and controls were drawn from the same distribution is now evaluated according to the Wilcoxon rank sum test. FDR selects frequencies 9-24-27 Hz with a false positive rate 0.05 . (c) FDR is applied to select separating electrodes, for $9 \mathrm{~Hz}$ flash stimuli. The vertical axis represent the probability that the $30 \Gamma_{s}$ values of patients and controls, for each sensor $s$, were drawn from the same distribution, according to the paired $t$ test. The labels for electrodes correspond to the international 10-20 system. (d) As in (c) for $24 \mathrm{~Hz}$ flash stimuli.

the pairs where $s$ is one the two sensors. For each frequency of stimuli, we apply the FDR method to select, among the 18 electrodes, those separating patients from controls according to their $\Gamma_{s}$. The results are depicted in Figs. 2(c) $(9 \mathrm{~Hz}$ case) and 2(d) $(24 \mathrm{~Hz})$ : eleven electrodes are recognized as separating in the case of $9 \mathrm{~Hz}$ stimuli and 13 in the case of $24 \mathrm{~Hz}$; no electrode is found to be individually separating when $27 \mathrm{~Hz}$ stimuli are considered. Since separating electrodes from all the regions of the cortex are found, it follows that the phenomenon here described is extended over all the cortex, not being localized in a limited region. Its diffuse nature suggests that genuine spatial synchronization [11] is here involved; indeed, volume conduction effects [12] would induce spatially more localized change.

Our data show that, for patients, the mean phase synchronization increases in the presence of visual stimuli, whereas it decreases in controls. For example, in the case of $24 \mathrm{~Hz}$ stimuli, and for all the sensors, the mean value (over subjects) of $\Gamma_{s}$ is shown in Fig. 3: hyper phase synchronization is observed in patients, whereas healthy subjects show a reduced phase synchronization. Similar patterns occur for 9 and $27 \mathrm{~Hz}$ stimuli. In Fig. 4 the histograms of the phase difference between electrodes 


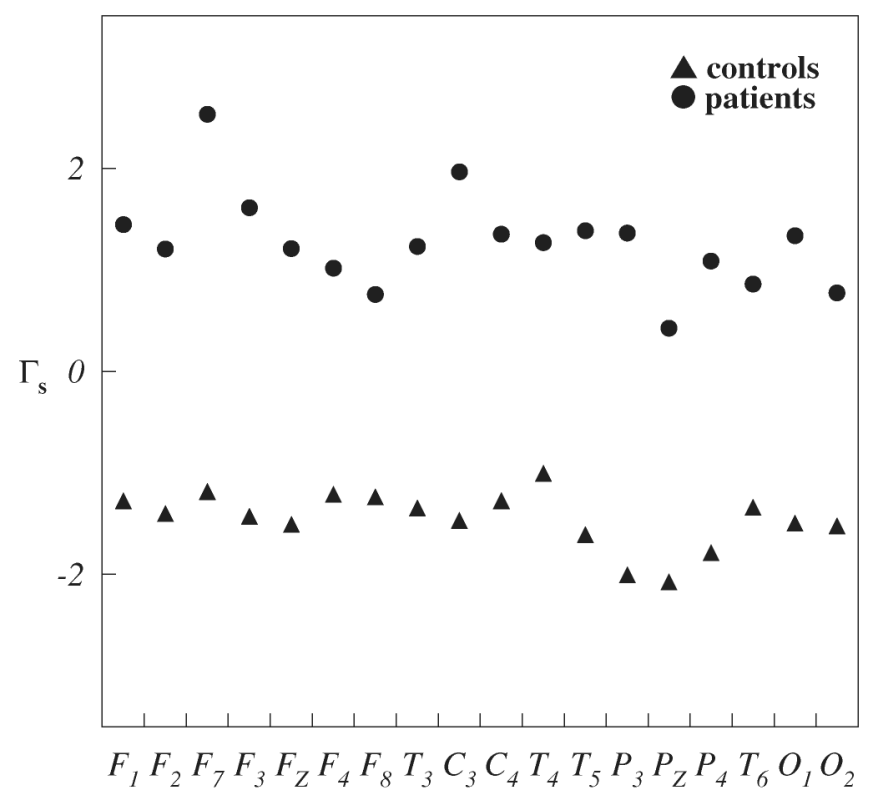

FIG. 3. In the case of $24 \mathrm{~Hz}$ stimuli, the mean of $\Gamma_{s}$ (over patients and over controls) is represented for all the 18 electrodes. On the average, phase synchronization increases for patients and decreases for controls.

T3 and T5, are shown for a migraine patient and for a control, both under stimulation and spontaneously. The distribution, when stimuli are delivered, broadens for the healthy person while becoming more peaked for the patient. This behavior is further illustrated in Fig. 5, where the time evolution of the phase difference between two sensors is depicted for a migraine patient, both subject to stimuli and in spontaneous conditions. In the presence of flash phase locking, in the two signals, is observed for time segments several seconds long; no such locking is observed in the spontaneous case. Phase difference curves, for a control, are also drawn in Fig. 5.
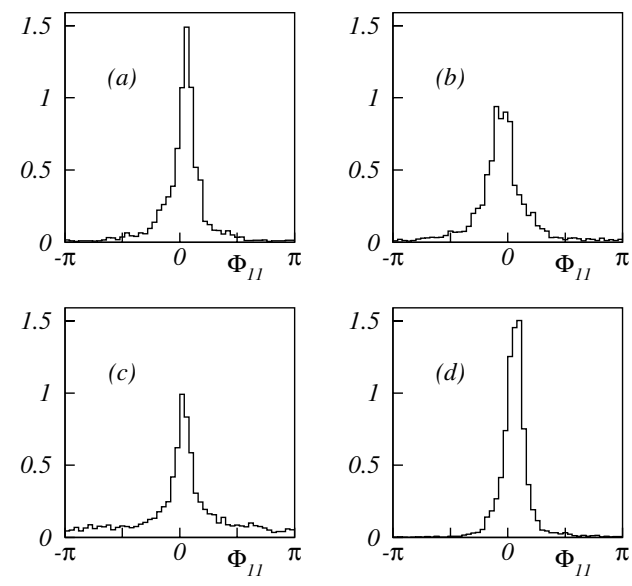

FIG. 4. Histogram of the phase difference for the pair T3-T5. (a) A healthy person without stimuli. (b) The same healthy person in presence of $9 \mathrm{~Hz}$ stimuli. (c) A patient without stimuli. (d) The same patient in presence of $9 \mathrm{~Hz}$ stimuli.
It is worth stressing that this phenomenon is not mined if coherence is used to measure synchronization: considering the linear index obtained by integration of the coherence function (normalized amplitude of the cross spectrum of the two time series [13]) in the alpha band, the corresponding $\Gamma$ and $\Gamma_{s}$ quantities do not lead to separation between patients and controls for any frequency of stimulation. We show that migraineurs are characterized by alpha band hypersynchronization in the presence of visual stimuli. We also show how this varies with the frequency of the flash, and present a topographic analysis where separating electrodes are recognized. Whilst it is comprehensible that $9 \mathrm{~Hz}$ stimuli might cause hypersynchronization in the alpha band (8$12.5 \mathrm{~Hz}$ ), in order to figure how $24-27 \mathrm{~Hz}$ stimuli may act on alpha oscillations we observe that brain is a nonlinear system, and subharmonics of $24-27 \mathrm{~Hz}$ fall in the alpha band: stimulation in the 24-27 band may cause hypersynchronization through their subharmonics. However, a similar behavior is not observed for other frequencies with subharmonics in the alpha band, like $18 \mathrm{~Hz}$ : further investigation is needed to clarify this aspect of the phenomenon. It will be also interesting to investigate the response of migraine patients with aura. Our results are consistent with current theories about the role of subcortical structures in migraine. Since the brainstem is active in migraine [14], it has been proposed, as a unifying concept of migraine, that brainstem regions concerned with the neural mechanism of synchrony are dysfunctional [15]. The cortex in migraine brains is thus misled by a dysfunctional gating system; normal light is unpleasant, normal sound uncomfortable, and, probably, normal pulsing of vessels felt as pain.

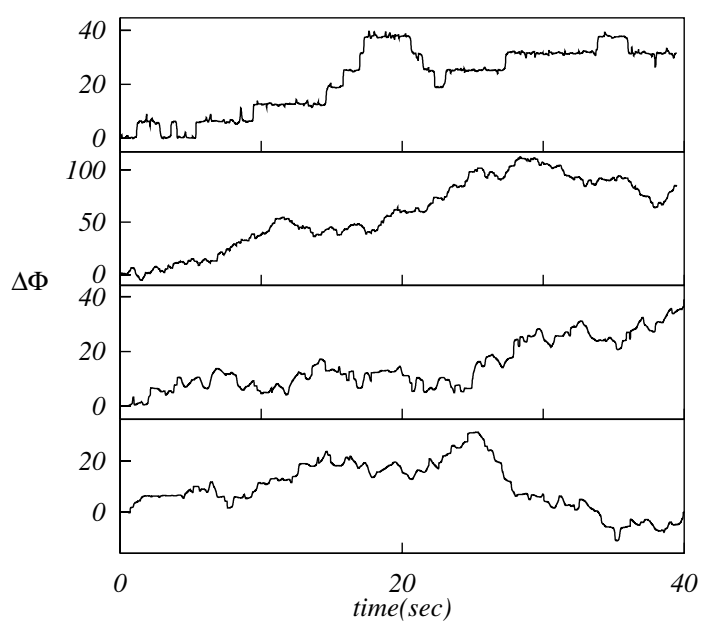

FIG. 5. Time evolution of $\Delta \Phi$ (i.e., the phase difference $\phi_{1}-$ $\phi_{2}$, without wrapping in the interval $[-\pi, \pi]$ ) for the pair F1F2. From the top to the bottom: for a migraine patient (the same patient as in Fig. 1) in the presence of $9 \mathrm{~Hz}$ stimuli; for the migraine patient without stimulation; for a control in presence of $9 \mathrm{~Hz}$ stimuli; for the control without stimulation. 
K. H. and P.Ch. I. acknowledge support from NIH (Grants No. R01 HL079046-01 and No. R01 HL07197203).

[1] M. G. Rosenblum et al., Phys. Rev. Lett. 76, 1804 (1996).

[2] C. Gray et al., Nature (London) 338, 334 (1989); M. Le Van Quyen et al., Lancet 357, 183 (2001).

[3] P. J. Goadsby et al., New England J. Med. 346, 257 (2002).

[4] V. Bohotin et al., Brain 125, 912 (2002); H. Bolay et al., Nat. Med. 8, 136 (2002); L. Battelli et al., Neurology 58, 1066 (2002).

[5] R. B. Silberstein, in Neocortical Dynamics and Human EEG Rhythms, edited by P. L. Nunez (Oxford University Press, New York, 1995), p. 272.

[6] There are two types of migraine, with and without aura. See, e.g., Headache Classification Committee of the International Headache Society, Cephalalgia 8, 1 (1988).
[7] P. Tass et al., Phys. Rev. Lett. 81, 3291 (1998); for an alternative approach using wavelets, see M. Le Van Quyen et al., J. Neurosci. Methods 111, 83 (2001).

[8] We consider $\ln (\gamma)$ instead of $\gamma$ because the distribution of $\ln (\gamma)$ values is found to be Gaussian, thus justifying use of $t$ test.

[9] J. Benjamini and Y. Hochberg, J. R. Stat. Soc. B 57, 289 (1995).

[10] The Bonferroni correction consists in lowering the threshold from 0.05 to $0.05 / N$, in the case of $N$ comparisons.

[11] R. Srinivasan, Clin. Neurophysiol. 110, 1351 (1999).

[12] Vol. conduction may be relevant for coherence estimates in high frequency scalp EEG. See, e.g., V. Menon et al., Electroencephalogr. Clin. Neurophysiol. 98, 89 (1996).

[13] Spectra are estimated by the Welch method, using half overlapped segments of 80 data points and a Hamming window. For a comparison between coherence and other synchronization measures, see R. Quian Quiroga et al., Phys. Rev. E 65, 41903 (2002).

[14] A. Bahara et al., Lancet 357, 1016 (2001).

[15] P. J. Goadsby, Cephalalgia 23, 565 (2003). 